\title{
Parent Report of Amplification Use in Children with Mild Bilateral or Unilateral Hearing Loss
}

DOI: $10.3766 /$ jaaa. 17020

\author{
Elizabeth M. Fitzpatrick*† \\ Isabelle Gabourył \\ Andrée Durieux-Smith*† \\ Doug Coyle§ \\ JoAnne Whittingham $\dagger$ \\ Mina Salamatmanesh*† \\ Rachel Lee* \\ Jessica Fitzpatrick 9
}

\begin{abstract}
Background: Amplification is considered to be one of the most important interventions for children with hearing loss. However, achieving consistent use of hearing technology in young children is an important problem, particularly when hearing loss is of mild degree. Little information is available about amplification use specifically for children with mild bilateral or unilateral hearing loss when such losses are targeted and identified early because of the availability of newborn hearing screening.
\end{abstract}

Purpose: We examined amplification use in a contemporary cohort of early-identified children with mild bilateral and unilateral hearing loss.

Research Design: As part of the Mild and Unilateral Hearing Loss in Children Study, we collected parent reports on their child's use of amplification during the preschool years.

Study Sample: A total of 69 children (38 unilateral and 31 bilateral mild) enrolled in the study from 2010 to 2015. Children entered the study at various ages between 12 and 36 mo of age and were followed up to age $48 \mathrm{mo}$. The median age of the children at enrollment was $16.5 \mathrm{mo}$ (interquartile range [IQR] $=9.5$, 26.8). Hearing loss was confirmed in these children at a median age of 3.6 mo (IQR $=2.4,5.7)$.

Data Collection and Analysis: Baseline characteristics related to the child and family were collected through an intake form at study enrollment. Data on amplification fitting and use were collected via parent questionnaires at each assessment interval. Information from parent questionnaires was summarized descriptively and amplification use was grouped into categories. Through logistic regression, we examined the relationship between amplification use and laterality of hearing loss, sex, and maternal education.

Results: Amplification was recommended for 59 (85.5\%) children at a median age of $6.5 \mathrm{mo}$ (IQR = 3.6, 21.2) and children were fitted at a median age of $10.9 \mathrm{mo}(\mathrm{IQR}=6.0,22.1)$. Based on parent report, hearing aid use was consistent for $39(66.1 \%)$ of 59 children who had amplification recommended. Parent questionnaires showed very little change in use for most of the children over the study period. More children with bilateral hearing loss used their amplification consistently than those with unilateral hearing loss. After adjusting for maternal education and sex of the child, the odds for consistent use in children with mild bilateral loss was almost seven times higher (odds ratio $=6.75 ; 95 \%$ confidence interval $=1.84$, 24.8) than for those with unilateral loss.

*Faculty of Health Sciences, University of Ottawa, Ottawa, ON, Canada; †Children's Hospital of Eastern Ontario Research Institute, Ottawa, ON, Canada; $\ddagger$ Department of Family Medicine and Emergency Medicine, Université de Sherbrooke, Sherbrooke, PQ, Canada; §School of Epidemiology, Public Health and Preventive Medicine, University of Ottawa, Ottawa, ON, Canada; $₫$ Dalla Lana School of Public Health Sciences, University of Toronto, Toronto, ON, Canada

Corresponding author: Elizabeth M. Fitzpatrick, Faculty of Health Sciences, University of Ottawa, Ottawa, ON K1H 8M5, Canada; Email: elizabeth. fitzpatrick@uottawa.ca

This study was funded through a Canadian Institutes of Health Research (CIHR, grant number 93705), and was supplemented by a CIHR New Investigator Award (2009-2014) and a Canadian Child Health Clinician Scientist Award (2009-2014) to the first author.

A brief preliminary section of this article was presented at the 33rd World Congress of Audiology in Vancouver, BC, Canada, September 21, 2016. 
Conclusions: Although $85.5 \%$ of children with mild bilateral or unilateral hearing loss received amplification recommendations, only two-thirds achieved consistent use by age 3-4 yr based on parent report. Children with mild bilateral loss were more likely to use amplification during the preschool years than those with unilateral loss.

Key Words: amplification, children, hearing aids, mild hearing loss, unilateral hearing loss

Abbreviations: $\mathrm{Cl}=$ confidence interval; $\mathrm{IQR}=$ interquartile range; $\mathrm{MUHL}=$ mild bilateral and unilateral hearing loss; OR $=$ odds ratio

\section{INTRODUCTION}

$\mathrm{T}$ he first intervention tool available for most children with permanent hearing loss is appropriate amplification. As proposed by Tomblin et al (2015), amplification use may be one of the important moderators of the negative impact of hearing loss on auditory and communication development outcomes. Although amplification is the standard care for children with moderate and greater hearing loss, decisions and follow-up for children with bilateral mild or unilateral loss (frequently referred to as minimal hearing loss) have been less certain (McKay et al, 2008; Fitzpatrick et al, 2010; 2014; Bagatto and Tharpe, 2014; Bagatto et al, 2016). There is a lack of evidence about the effectiveness and use of hearing aids or any intervention for these children, resulting in some universal hearing screening programs setting the target definition for screening at moderate hearing loss (Wood et al, 2015). However, in programs where mild hearing loss is targeted, the age of diagnosis has been substantially reduced from school age to $<1$ to $2 \mathrm{yr}$ of age (Fitzpatrick et al, 2014; Ghogomu et al, 2014), leading to earlier intervention with amplification.

The problems related to mild bilateral and unilateral hearing loss (MUHL) from a hearing perspective and consequently, the reasons for consideration of amplification are different. Unilateral loss results in a binaural hearing deficit, which leads to difficulty with localization and listening in noise, whereas mild bilateral loss results in a reduction in access to soft speech, which also affects speech understanding particularly in the presence of noise. Despite these fundamental differences in the disorders, many of the problems children experience academically and in communication as well as the challenges relative to amplification use reported in the literature are remarkably similar (Porter et al, 2016). In our qualitative interviews with parents of preschool children, which included 11 with unilateral and 9 with mild bilateral loss, parents' discussions of the challenges, doubts, and questions around the need for and use of amplification were strikingly similar (Fitzpatrick et al, 2016).

Although guidelines for pediatric amplification are well-established for hearing loss of moderate degree and greater (King, 2010; AAA, 2013; Bagatto et al, 2016), there is some uncertainty about the benefits of amplification for children with mild loss or unilateral loss. This uncertainty results frequently in consideration of hearing aids on a case-by-case basis for these children (McKay et al, 2008; Bagatto and Tharpe, 2014). The overwhelming majority of children with milder losses are eventually fitted with hearing aids although the gap between diagnosis and amplification recommendation is longer than for those with more severe loss (Fitzpatrick et al, 2010; 2014; Walker, Holte, et al, 2015). Furthermore, considerable clinical indecision and inconsistent hearing aid use have been reported, suggesting nonuse or inconsistent use in 30 $50 \%$ of these children (Davis et al, 2001; Reeve, 2005; Fitzpatrick et al, 2010; 2014). However, most of these data are from children who did not undergo newborn hearing screening and were identified later in childhood. More recent data from a longitudinal study that included results for 38 children with mild bilateral loss showed that about $37 \%$ of the children used their hearing aids on a full-time basis ( $>8.3 \mathrm{hr}$ per day), $40 \%$ were part-time users, and $23 \%$ did not use them (Walker, Holte, et al, 2015). There seems to be a tendency to amplify the overwhelming majority of children with mild bilateral or unilateral loss eventually; that is, there are perceived benefits from amplification (Fitzpatrick et al, 2017). However, further research is needed to determine the advantages of amplification for this clinical population and to assist in establishing best practices.

Recent research investigating hearing aid use either through data logging in digital hearing aids or using parent report has drawn attention to the challenges in achieving consistent amplification use in young children across the full spectrum of severity of hearing loss (Jones and Launer, 2011; Walker et al, 2013; Muñoz and Hill, 2015; Muñoz et al, 2015; Walker, McCreery, et al, 2015). Jones and Launer (2011) reported an average of $5.5 \mathrm{hr}$ per day of hearing aid use based on objective data logging records for approximately 5,000 children from birth to $19 \mathrm{yr}$ of age in the United States with $40 \%$ of children in the $0-4 \mathrm{yr}$ age range achieving $<4 \mathrm{hr}$ daily hearing aid use. More recently, Muñoz et al (2015), in a survey of 37 families of young children at a mean age of $22 \mathrm{mo}$, found that parents reported $<5 \mathrm{hr}$ of daily hearing aid use for $31 \%$ of children. Using both data logging and parent report data, Walker, McCreery, et al (2015) found substantially higher average hours of use of $10.6 \mathrm{hr}$ (parent report) and $8.4 \mathrm{hr}$ (data logging) per day for 290 children (age 6 mo to $7 \mathrm{yr}$ at start of study) followed in a longitudinal study. The preschool children with visits documented from 2 to $4 \mathrm{yr}$ of age had an average of 7.6 parent-reported hours 
of amplification use and $79 \%$ wore their hearing aids $>8 \mathrm{hr}$ daily over the study (up to $6 \mathrm{yr}$ of age).

There is also evidence that hearing aid use varies widely depending on factors such as the listening context, age of the child, degree of hearing loss, and socioeconomic status (Moeller et al, 2009; Walker et al, 2013; Walker, McCreery, et al, 2015; Muñoz et al, 2016). In children with mild to moderate hearing loss, Moeller et al (2009) found that only two of seven parents reported that they established consistent hearing aid use in multiple listening environments before $2 \mathrm{yr}$ of age. Similarly, Muñoz et al (2015) reported that car rides were particularly problematic for parents. In some studies, children with milder degrees of hearing loss showed greater vulnerability to less hearing aid use (Walker et al, 2013; Muñoz et al, 2014; 2016). For example, in a 2013 study on the same cohort described earlier $(\mathrm{n}=$ 272 , mean age of $40.51 \mathrm{mo}$ ), Walker et al (2013) found that more hearing aid use was related to older age, poorer hearing, and higher maternal education level. Children with hearing loss $<50 \mathrm{~dB}$ HL used hearing aids about one hr less daily (9.95 compared with 11.12 parent-reported hours per day) than those with $>50 \mathrm{~dB}$ loss. However, in their 2015 study, an examination of factors affecting hearing aid use trends did not show any association with severity of hearing loss or maternal education for the preschool group $(\mathrm{n}=$ 125; Walker, McCreery, et al, 2015).

Taken together, these studies point to the challenges associated with early amplification use, which is an essential component of intervention in pediatric hearing care. However, there is still relatively limited information about the specific population of children with mild hearing loss or unilateral loss and their amplification use in the current context of screening and early intervention. Yet, combining mild bilateral and unilateral loss, these children now account for $40-50 \%$ of children identified with permanent hearing loss during the preschool years (Fitzpatrick et al, 2014; Barreira-Nielsen et al, 2016). The purpose of this study was to examine hearing aid use in a contemporary cohort of children who were diagnosed with mild bilateral or unilateral hearing loss following the implementation of newborn hearing screening. We also sought to identify factors that influence hearing aid use.

\section{METHODS}

\section{Design and Setting}

The MUHL study is a multicenter longitudinal cohort study investigating developmental outcomes in preschool-age children with mild bilateral or unilateral hearing loss. As part of the study, we collected information from parents on amplification recommendations and use.
Definitions for mild and unilateral hearing loss for this study were adapted from the National Workshop on Mild Bilateral and Unilateral Hearing Loss (2005): mild bilateral hearing loss-average pure-tone air conduction thresholds (at $0.5,1$, and $2 \mathrm{kHz}$ ) between 20 and $40 \mathrm{~dB}$ HL or thresholds $>25 \mathrm{~dB}$ HL at two or more frequencies greater than $2 \mathrm{kHz}$; unilateral hearing loss-hearing loss in one ear only with a pure-tone average $\geq 20 \mathrm{~dB}$ HL or $>25 \mathrm{~dB}$ at two or more frequencies greater than $2 \mathrm{kHz}$. In this study, we categorized children as mild bilateral based on hearing levels in the better ear.

In 2002, a province-wide universal newborn hearing screening program, which involves screening, early communication development, and parent support was implemented in the province of Ontario, Canada, a province of approximately 11 million people (Hyde et al, 2004). The program includes MUHL in the target disorder. In the Ontario program, children referred from screening undergo diagnostic assessment at a designated pediatric audiology program. Children who have hearing loss confirmed are seen by an otolaryngologist to confirm permanent hearing loss and to receive medical clearance for hearing technology, if applicable. Parents make an informed choice about hearing technology and other intervention options such as therapy (Brown and Mackenzie, 2005). Children continue audiological follow-up at 3 and 6 mo intervals, respectively, during the first and second year of amplification followed by annual visits for the first $6 \mathrm{yr}$ (OMCYS, 2014). Frequency of therapy is determined by the child's intervention program. All services are publicly funded.

\section{Participants}

Inclusion criteria for enrolment in the MUHL study included (a) less than age $3 \mathrm{yr}$ at study enrollment, (b) permanent mild bilateral (better ear) or unilateral hearing loss, (c) congenital or early onset hearing loss (before age $6 \mathrm{mo}$ ), and (d) English as one of the languages spoken at home. Children with auditory neuropathy spectrum disorder were excluded from the study as well as those with a diagnosis of severe developmental delay based on the requirement to complete multiple spoken language assessments.

Families were enrolled from 2010 to 2015 in the longitudinal project and were recruited through their clinical providers from three regions in Ontario (Ottawa, Toronto, and Southern Ontario). Recruitment took place at several different sites, and we were unable to collect information on how many families were approached. Children entered the study at various ages between 12 and 36 mo and were followed up to age $4 \mathrm{yr}$. The study received ethics approval from the Children's Hospital of Eastern Ontario (\#09-64×) Research Institute (main study site) and the University of Ottawa (\#H10-09-11), and met ethics requirements for all of the clinical programs that invited families to participate in the study. 


\section{Study Procedures}

For this report, our focus is on amplification information collected through parent-completed questionnaires. For the study, baseline characteristics related to the child and family were collected through an intake form at study enrollment. Families were asked to sign a consent form so that details about the diagnosis and degree of hearing loss as well as amplification recommendations and fitting could be collected from the child's audiology program. At enrolment, families were also sent a study-specific intervention questionnaire with questions about amplification recommendations, amplification use, hearing health, and therapy services (Supplemental Appendix S1). This questionnaire was resent to the families at each annual assessment and they were invited to update the information between each assessment. Audiogram information from the child's clinical program was examined to document any change in hearing over the course of the study and to confirm parent reports of recommendations for amplification.

For this part of the study on amplification, data were extracted from the intervention questionnaire related to the section entitled "Your Child's Amplification," which included three questions on amplification recommendations and use (Supplemental Appendix S1). As shown in the questionnaire, parents were specifically asked to report whether their child had amplification recommended (yes, no) and whether it was used (yes, no), and to identify the amount of use by checking one of the four responses provided: all day (all waking hours); most of the time ( $>6 \mathrm{hr})$; not often $(<6 \mathrm{hr})$; and never. These categories were somewhat arbitrary and based primarily on the notion that this study involved young early-identified children and that, after accounting for naptime, $>6 \mathrm{hr}$ was a reasonable estimate for "most of the time." Data logging information was not captured during the study. For this observational study, no protocol was in place to check or modify the child's hearing instrument(s). As part of the Ontario Infant Hearing Program protocol, children are followed by a designated audiology clinic with trained providers who are required to follow the Infant Hearing Program protocol for fitting and management of amplification (OMCYS, 2014).

Information from parent reports was collapsed into four categories to capture amplification use over the time of the study:

- Consistent use-children with a recommendation for amplification and consistent use (all day, most of the time) over the course of the study.

- Inconsistent use-children with a recommendation for amplification and limited use (not used some of the time, not often used) over the course of the study.
- Not used-children with a recommendation for amplification and not used at all during the study.

- Not recommended-parents reported no recommendation for amplification.

\section{Data Analysis}

Statistical analysis was performed using SPSS Version 24 (IBM Corporation, Armonk, NY). Baseline characteristics for the participants were summarized with descriptive statistics and included frequency counts as well as means and standard deviations or medians and interquartile ranges, as appropriate.

Parents' report of amplification use during the study was first examined descriptively and categorized according to parent questionnaire responses into one of four categories: consistent use, inconsistent use, not used, or not recommended. We also explored descriptively the pattern of hearing aid use for children who had at least two completed questionnaires $(\mathrm{n}=$ 58) to monitor any change in hearing aid use.

Using logistic regression, we estimated the association between child or family factors and amplification use. For this analysis, the response categories for those with hearing aids recommended were first collapsed into two broader options, (a) most or all day use (category 1 as described earlier) and (b) little or no use (categories 2, 3 described earlier). Univariate analyses were first carried out to examine the association between hearing aid use and the following variables: laterality (bilateral versus unilateral), sex, type of hearing loss (sensorineural or permanent conductive), age at diagnosis $(<6$, $\geq 6 \mathrm{mo}$ ), maternal education, and home language. Because of their association with the variable of interest, laterality, sex, and maternal education were entered into the regression model. This number of predictors should allow for the estimation of a valid model (Peduzzi et al, 1996). Using $\chi^{2}$ analysis, we also explored whether there was an association between degree of hearing loss in the worse ear (mild versus $>$ mild) for the bilateral group and in the impaired ear (mild versus $>$ mild) in the unilateral group and having amplification recommended or not. Significance was accepted at the $p=$ 0.05 level and all $p$-values were two-sided.

\section{RESULTS}

\section{Clinical Characteristics of Participants}

The results reported in the later paragraphsare based on a total of $184(83.6 \%)$ questionnaires received over the course of the study for the 69 children (36 boys and 33 girls) in the study. Based on age at enrolment, a total of 220 questionnaires would have been expected. However, eight families withdrew before study end (12 
questionnaires), seven children were younger than 48 mo at study end (nine questionnaires), and 15 other questionnaires were not returned at annual follow-up, thus $83.6 \%$ (184 of 220 ) of the possible questionnaires were received. A total of 11 parents provided one questionnaire, 18-two questionnaires, 23-three questionnaires, and 17-four questionnaires. In addition, a total of 153 audiology reports were received with at least one report for each child.

Detailed clinical characteristics of the children are reported in Table 1 for the full group as well as individually according to mild bilateral or unilateral loss. The sample consisted of 38 (55.1\%) children with unilateral and $31(44.9 \%)$ with bilateral hearing loss at initial diagnosis. The families who agreed to participate in the study tended to have completed postsecondary education; mean maternal education level was $17.4 \mathrm{yr}$ (standard deviation $=3.5$ ). As shown, this was an early-identified cohort with $91.3 \%$ (63 of 69) of children having undergone screening. Children were identified at a median age of 3.6 (interquartile range $[\mathrm{IQR}]=2.4,5.7$ ) $\mathrm{mo}$. The majority of the children $(85.5 \%, \mathrm{n}=59)$ presented with congenital loss and an additional four (5.8\%) children had known early onset hearing loss (before age $6 \mathrm{mo})$. A total of $75.4 \%(\mathrm{n}=52)$ had sensorineural loss. The relatively large number of children with permanent conductive loss $(24.6 \%, \mathrm{n}=17)$ is consistent with ear, nose, and throat malformations (microtia and atresia) as the single largest known etiology, accounting for $21.7 \%$ of known etiologies. Of the 38 children

Table 1. Characteristics of 69 Children with Mild Bilateral or Unilateral Hearing Loss

\begin{tabular}{|c|c|c|c|}
\hline Characteristics & Unilateral HL & Mild Bilateral HL & All \\
\hline $\mathrm{N}$ & 38 & 31 & 69 \\
\hline Sex (male \%) & $23(60.5 \%)$ & $13(41.9 \%)$ & $36(52.2 \%)$ \\
\hline Maternal education, \# yr, mean (standard deviation)* & $17.2(3.4)$ & $17.7(3.6)$ & $17.4(3.5)$ \\
\hline \multicolumn{4}{|l|}{ Screening status } \\
\hline Screened & $35(92.1 \%)$ & $28(90.3 \%)$ & $63(91.3 \%)$ \\
\hline Not screened or unknown status & $3(7.9 \%)$ & $3(9.7 \%)$ & $6(8.7 \%)$ \\
\hline Age confirmation, mo, median (IQR) & $3.4(2.0,5.5)$ & $3.6(2.7,5.9)$ & $3.6(2.4,5.7)$ \\
\hline Age enrolment, mo, median (IQR) & $14.2(9.0,26.4)$ & $19.3(10.8,28.1)$ & $16.5(9.5,26.8)$ \\
\hline Age final assessment, mo, median (IQR) & $47.8(38.8,48.5)$ & $48.1(47.2,49.3)$ & $48.0(46.2,48.6)$ \\
\hline \multicolumn{4}{|l|}{ Onset of hearing loss, $\mathrm{n}(\%)$} \\
\hline Congenital & $35(92.1 \%)$ & $24(77.4 \%)$ & $59(85.5 \%)$ \\
\hline Early onset (<6 mo) & 0 & $4(12.9 \%)$ & $4(5.8 \%)$ \\
\hline Late onset (>6 mo) & $2(5.3 \%)$ & $1(3.2 \%)$ & $3(4.3 \%)$ \\
\hline Unknown & $1(2.6 \%)$ & $2(6.5 \%)$ & $3(4.3 \%)$ \\
\hline \multicolumn{4}{|l|}{ Type of hearing loss, $\mathrm{n}(\%)$} \\
\hline Sensorineural & $24(63.2 \%)$ & $28(90.3 \%)$ & $52(75.4 \%)$ \\
\hline Conductive & $14(36.8 \%)$ & $3(9.7 \%)$ & $17(24.6 \%)$ \\
\hline \multicolumn{4}{|l|}{ Etiology known } \\
\hline Neonatal intensive care unit & $2(5.3 \%)$ & 0 & $2(2.9 \%)$ \\
\hline Hereditary/genetic & $2(5.3 \%)$ & $6(19.4 \%)$ & $8(11.6 \%)$ \\
\hline Syndromes & $1(2.6 \%)$ & $2(6.5 \%)$ & $3(4.3 \%)$ \\
\hline ENT malformations & $13(34.2 \%)$ & $2(6.5 \%)$ & $15(21.7 \%)$ \\
\hline Cytomegalovirus & $1(2.6 \%)$ & $1(3.2 \%)$ & $2(2.9 \%)$ \\
\hline Etiology unknown & $19(50.0 \%)$ & $20(64.5 \%)$ & $39(56.5 \%)$ \\
\hline \multicolumn{4}{|l|}{ Degree of hearing loss at diagnosis (impaired/worse ear) } \\
\hline High frequency $\dagger$ & 0 & $5(16.1 \%)$ & $5(7.2 \%)$ \\
\hline Mild (20-40 dB HL) & $3(7.9 \%)$ & $19(61.3 \%)$ & $22(31.9 \%)$ \\
\hline Moderate (41-55 dB HL) & $7(18.4 \%)$ & $4(12.9 \%)$ & $11(15.9 \%)$ \\
\hline Moderately severe (56-70 dB HL) & $13(34.2 \%)$ & $3(9.7 \%)$ & $16(23.2 \%)$ \\
\hline Severe $(71-90 \mathrm{~dB} \mathrm{HL})$ & $11(28.9 \%)$ & 0 & $11(15.9 \%)$ \\
\hline Profound (>90 dB HL) & $4(10.5 \%)$ & 0 & $4(5.8 \%)$ \\
\hline Age rec amplif, mo, median (IQR)末 & $6.7(4.6,30.0)$ & $4.9(3.2,14.9)$ & $6.5(3.6,21.2)$ \\
\hline Age fitting amplif, mo, median (IQR)§ & $12.2(7.2,29.9)$ & $10.5(5.2,21.7)$ & $10.9(6.0,22.1)$ \\
\hline
\end{tabular}

amplif = amplification; ENT = ear nose throat (anomalies included atresia and microtia); HL = hearing loss; rec = recommendation. *Not reported by three families.

$\dagger$ Defined as $\geq 25 \mathrm{~dB} \mathrm{HL}$ at $\geq 2$ frequencies above $2 \mathrm{kHz}$.

\$Age was available for 27 of 30 children with unilateral hearing loss and for 27 of 29 with mild bilateral loss who received amplification recommendations.

$\S$ Age of fitting was available for 21 of 26 children with unilateral hearing loss and for 25 of 27 with mild bilateral loss. 
with unilateral loss, $23(60.5 \%)$ had $<70 \mathrm{~dB}$ loss in the impaired ear at diagnosis, whereas $15(39.5 \%)$ presented with severe or profound loss. For the 31 children with bilateral loss, degree of hearing loss in the worse ear at diagnosis ranged from high frequency only to $<70 \mathrm{~dB}$, with the majority presenting with mild loss $(61.3 \%, \mathrm{n}=19)$. Based on audiological reports, six children showed deterioration in hearing at some point during the study.

\section{Overall Amplification Use}

Figure 1 shows an overall summary of amplification recommendations and use for children with bilateral and unilateral hearing loss. Overall, 59 of the 69 children $(85.5 \%)$ received a recommendation for amplification based on parent and audiologist reports at diagnosis or during the study. This included 30 of 38 $(79.0 \%)$ children with unilateral loss and 29 of 31 (93.6\%) with bilateral loss. Amplification was recommended at an early age at a median of 6.5 mo (IQR = $3.6,21.2)$. A total of $53(89.8 \%)$ of the children who received a recommendation were fitted with amplification (one with frequency modulation system only) at a median age of $10.9(6.0,22.1)$ mo. Parents of an additional six children with recommendations for amplification reported that they did not acquire it.

Overall, $39(66.1 \%)$ of the 59 children who received amplification recommendations acquired and achieved consistent use in the preschool years, whereas the remaining $20(33.9 \%)$ had inconsistent use $(n=7)$ or did not use or acquire amplification at all $(n=13)$. A substantially larger proportion of children with mild bilateral loss ( 25 of the $29,86.2 \%$ ), who had amplification recommended, used it consistently compared with 14 of the $30(46.6 \%)$ of those with unilateral loss. Although recommended, amplification was not used at all by 11 of the 38 children (28.9\%) with unilateral loss and 2 of the 29 (6.9\%) with bilateral loss.

\section{Trends in Amplification Use}

We qualitatively examined trends in hearing aid use over time for 58 children whose parents provided at least two questionnaires. By the study end, 36 $(62.1 \%)$ of these had achieved consistent use and the remaining $22(37.9 \%)$ continued to show inconsistent or no use based on parent report. In the consistent use group, as shown in Figure 2, increases in amplification use were seen over time for 8 of the $36(22.2 \%)$ children. These were eight children whose parents, at the start of the study (first questionnaire), reported no use $(\mathrm{n}=3)$ or not often used $(\mathrm{n}=5)$. In the inconsistent use group, only one parent reported a change in use over time, decreasing from most of the time at $24 \mathrm{mo}$ of age to not often at age 36 and $48 \mathrm{mo}$. Similarly, in those who had no amplification use by the study end, no changes were observed over time except for one child who showed variation across four questionnaires, moving from "not used" to "most of the time" to "not often" to "not used."

\section{Factors Affecting Amplification Use}

There was no significant difference in consistency of amplification use based on sex (odds ratio $[\mathrm{OR}]=0.52$; $95 \%$ confidence interval $[\mathrm{CI}]=0.15,1.75)$ or maternal education $(\mathrm{OR}=1.18 ; 95 \% \mathrm{CI}=0.76,1.84)$. However, compared with children with unilateral hearing loss, those with bilateral loss were more likely to use amplification consistently, that is, they had 6.75 times the odds of consistent use $(\mathrm{OR}=6.75 ; 95 \% \mathrm{CI}=1.84,24.8)$.

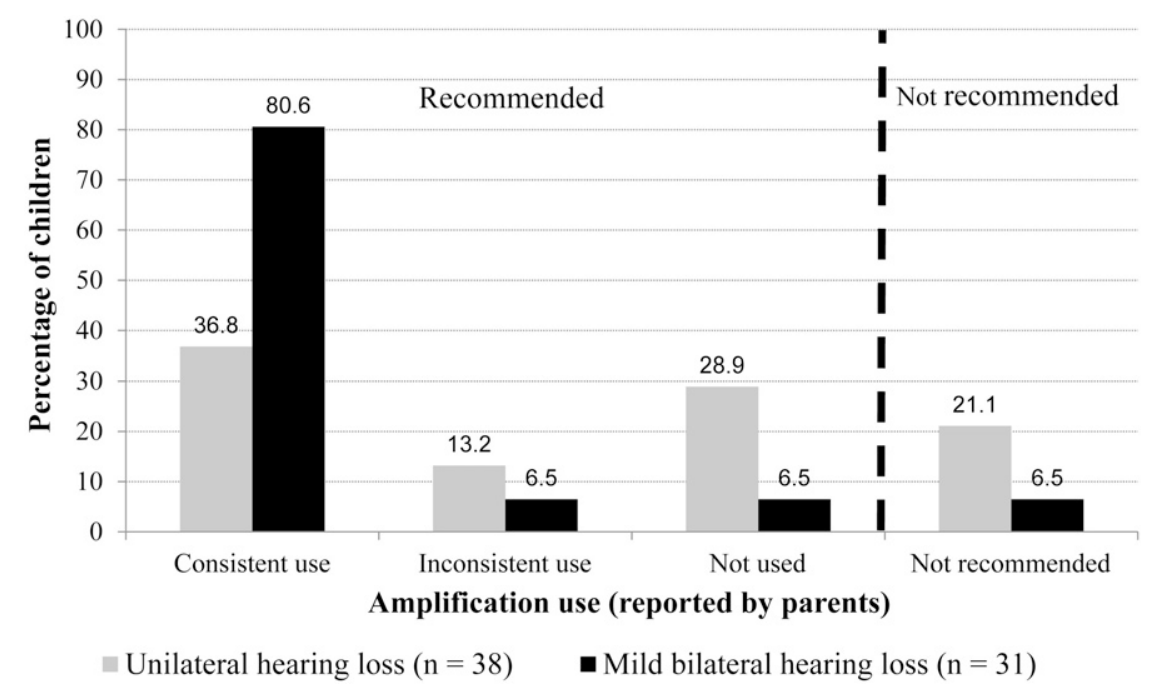

Figure 1. Amplification recommendations and use for 69 children based on parent report. 


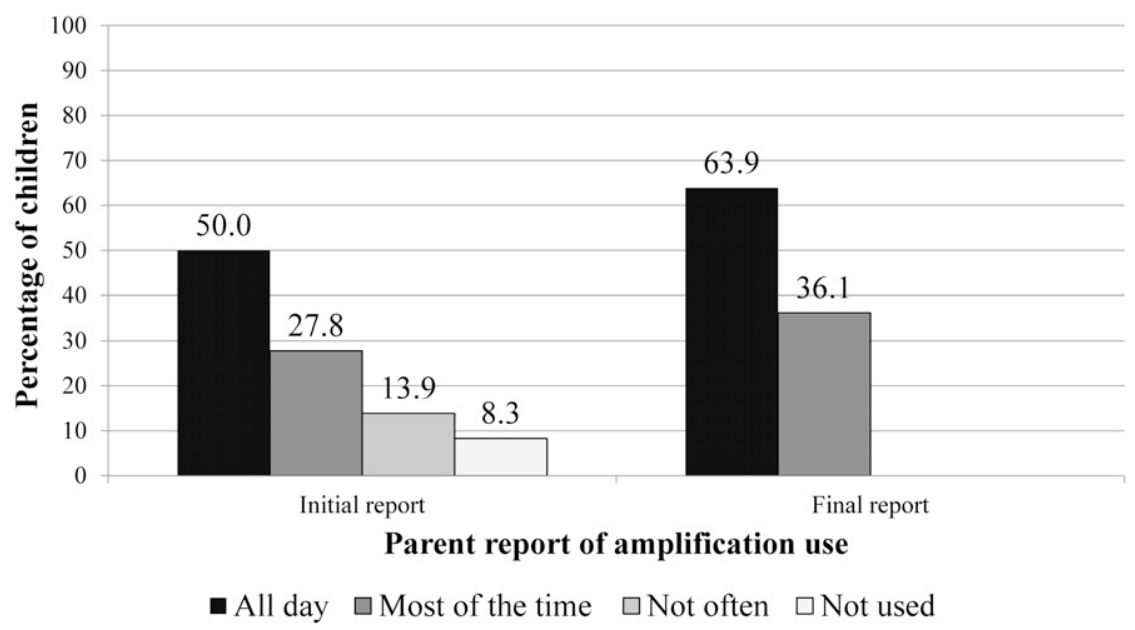

Figure 2. Change in amplification use from initial to last parent report for children who achieved consistent use ( $\mathrm{n}=36$ ).

We also explored the effect of degree of hearing in the worse ear related to hearing aid use for the 31 children with mild bilateral loss because poorer hearing in the worse ear was associated with hearing aid recommendations in our previous research (Fitzpatrick et al, $2010 ; 2014)$. There was, however, no significant association with degree of hearing loss $(p=0.38)$ in the present study. An exploration of the association between having amplification recommended (yes, no) and degree of hearing loss in the worse ear (mild bilateral group) or degree in the impaired ear (unilateral loss group) showed no significant difference for either group (Fisher's exact test: $p=1.0$ for both groups).

\section{DISCUSSION}

$\mathrm{E}$ arly identification of large numbers of children with MUHL is a relatively recent consequence of the widespread implementation of newborn screening programs. Our findings showed that clinicians overwhelmingly recommended amplification for these children regardless of degree or laterality of hearing loss, with $85.5 \%$ of all children receiving recommendations for amplification by the end of our study. Furthermore, on average, amplification was recommended for these early-identified children at much earlier ages (on average 6.5 mo of age) than previously documented (Durieux-Smith et al, 2008; Fitzpatrick et al, 2014). However, 1 in 5 children with unilateral hearing loss did not have any type of amplification prescribed compared with just 1 in 15 of children with mild bilateral loss. Although amplification was widely recommended, just two-thirds of parents reported that consistent use had been established in the preschool years. The problem was particularly concerning for children with unilateral loss because although amplification was recommended for $79 \%,<50 \%$ (14 of 30) achieved consistent device use. More than one-third of those with unilateral loss, who had received recommendations for amplification, reported not having acquired or used a hearing instrument. By contrast, the majority $(86.2 \%, 25$ of 29$)$ of children in the bilateral group consistently used their amplification and only two families reported total nonuse. An examination of trends in use for all children suggested that most of the parents either adopted amplification and used it from the beginning or achieved little or no use over time. For most children, there was little increase or decrease in the amount of use reported across the study period.

This study adds to the growing body of knowledge (Martin et al, 2005; Moeller et al, 2009; Walker et al, 2013; Muñoz and Hill, 2015; Muñoz et al, 2015; Walker, McCreery, et al, 2015) that has brought greater awareness to the challenges for families in establishing early amplification use. Of particular importance, this study adds information specific to an early-identified cohort of children with minimal hearing loss. Comparison with other studies is somewhat challenging as there are variations in definitions/cut-points used to define consistency of use. Our overall study results, with one-third of children showing inconsistent or no amplification use, are similar to those of Muñoz et al (2015) where $31 \%$ of parents of 37 children (unilateral and bilateral mild-to-severe loss) reported $<5 \mathrm{hr}$ of daily hearing aid use. It is noteworthy that parent-reported use for children with "minimal" losses in our study was remarkably similar to those for children across the spectrum of hearing loss. Interestingly, in contrast to our findings, Muñoz et al (2014) found that children (age = 7 mo to $6 \mathrm{yr})$ with unilateral loss $(\mathrm{n}=7)$ used their hearing aid more hours than those with mild loss in the better ear $(\mathrm{n}=10)$.

Although Walker et al (2013) and Walker, McCreery, et al (2015) found higher parent-reported hearing aid use overall (approximately $10 \mathrm{hr}$ daily) than the aforementioned studies, children with $<50 \mathrm{~dB}$ loss used 
amplification less consistently. In previous research, we found that greater degree of hearing loss in the worse ear for children with bilateral loss was related to audiologists' decisions to amplify (Fitzpatrick et al, 2010; 2014). However, in the present study, we did not find that having greater than mild loss in the worse ear affected consistency of use for the 31 children with bilateral loss. It is possible that the younger age of children in this study and, therefore, shorter time with hearing aids may have contributed to these different findings. Another possibility is that clinical practices including counseling have changed and that audiologists strongly encourage parents to use their child's hearing aids regardless of degree of hearing loss. Amplification was recommended for a larger proportion of children and with a shorter gap between diagnosis and fitting than we have documented in previous research with older identified children (Fitzpatrick et al, 2010; 2014).

Our findings are also aligned with those of Moeller et al (2009) who reported hearing aid use to be highly variable in early-identified children with mild to moderately severe hearing loss. Our findings did not, however, concur with these investigators' results that on average, amplification use became more consistent during the second year of life. Walker, McCreery, et al (2015), who collected information on use from both parent report and data logging values, also found that most of the 290 children with bilateral mild-to-severe hearing loss increased use over time, although a minority also decreased use. Likewise, Muñoz et al (2014) also reported that hearing aid use increased with age (age $=7 \mathrm{mo}$ to $7 \mathrm{yr}$ ) as well as with more severe hearing loss. For most of the children, we did not observe a change in the pattern of use over the course of our study, but these children had only reached a maximum age of 48 mo by the end of the study.

It is important to note that our study population was different from that described in the reports mentioned earlier as we included only children with mild bilateral or unilateral hearing loss. It is possible that the auditory benefits of hearing aids are not as readily observable in these children's spoken language acquisition and auditory behavior (Walker, Holte, et al, 2015). Most families who reported use may be those who simply "buy in" from the beginning. Indeed, interviews reported in a previous study with a subset of 20 families from our study (Fitzpatrick et al, 2016) revealed that decisionmaking and using hearing aids was one of the most confusing experiences for families. Walker, McCreery, et al (2015) found that maternal education influenced trends in hearing aid use. In our study, we did not detect any significant relationship between maternal education and parent report of amplification use. However, the small sample size and the fact that most mothers were university educated may have precluded this finding. Population-based studies that examine use in children across a broader spectrum of sociodemographic status may be able to shed light on the association between hearing aid use and parenteducation level.

Considered in the context of reports on large datasets for children across the spectrum of hearing loss, which showed that $40 \%$ of children used hearing aids $<40 \%$ of the time (Jones and Launer, 2011; Jones and Feilner, 2013), our results may at first appear encouraging. Based on parent report, two-thirds of these young children with mild bilateral hearing loss used amplification $>6 \mathrm{hr}$ per day. However, as reported by Walker et al (2013), data logging showed that parents seriously underestimated the actual amount of use. Walker, Holte, et al (2015) concluded that improved audibility through full-time or part-time hearing aid use was associated with better language scores for 38 children (assessed at age 5 or $7 \mathrm{yr}$ ) with bilateral slight or mild loss. It is difficult to directly compare our results on amplification use to this study for several reasons. First, we used different cutoffs to categorize children. The Walker, Holte, et al (2015) study showed that about a third (37\%) of children achieved full-time use, but they used a cutoff of $>8.7 \mathrm{hr}$, whereas we categorized consistent use as $>6 \mathrm{hr}$ use. Furthermore, their documentation of hearing aid use was based mostly on data logging measures, and we depended solely on parent report. Finally, our study was focused on early-identified children (median age of $3.6 \mathrm{mo}$ ). By contrast, 15 of 38 children in the Walker, Holte, et al (2015) study were late-identified at an average age of $45.5 \mathrm{mo}$. Both these studies suggest that audiologists recommended amplification for these children, based on the assumption or measurement of improved audibility. This work, therefore, continues to point to the need for parent support in helping them to understand the need for hearing aids and for establishing consistent use with their young children.

A strength of our study is that it provides information on a large group of early-identified children with minimal loss followed prospectively over several years. It is also an advantage that children were serviced in different clinical centers within the same province; therefore, providing a broader representation of this clinical population. However, like other longitudinal studies of this type, which require voluntary and long-term commitment, our research tends to be biased toward families of higher socioeconomic status. Therefore, the findings may not be as readily applicable to families from more diverse socioeconomic backgrounds.

The study is limited to a broad parent report of hearing aid use as we did not collect parent report details on hearing aid use across specific environments such as in the car, in playgroups and in daycare settings. Furthermore, no comparative objective measurement of amplification use is available as data logging information was not collected because the study protocol was planned before widespread availability and application of this option. Previous studies have reported high (0.76) 
correlations between data logging and parent report (Walker et al, 2013; Walker, McCreery, et al, 2015), although $84 \%$ of parents overestimated use by an average of $>2.3-2.6 \mathrm{hr}$ (Walker, McCreery, et al, 2015). This finding suggests that parent report of use is a reasonably reliable measure despite overestimation of the actual amount of time hearing instruments are worn. Our MUHL study was an observational study whose primary purpose was to examine auditory and communication development outcomes in early-identified children. We did not collect information on the quality of hearing aid fitting or care. As noted previously, all children with amplification were required to be followed according to protocols established by the publicly funded provincial program (OMCYS, 2014).

The relatively few changes observed over time for children suggest that most parents, either adopted amplification and used it consistently from the beginning or simply did not understand or otherwise "buy into" the need for amplification. We were not able to collect sufficient reliable information about the type and amount of intervention to glean insights into whether therapy or particular audiological support early in the intervention process influenced parents' decisions to use the prescribed amplification. Further research could help determine whether greater investment from audiology and early intervention programs in supporting parents to use the amplification could make a difference. At a minimum, our research suggests that the majority of parents are unlikely to change their habits related to amplification practices over time. This finding connects with our previous qualitative research with a subset of parents from this study who shared their misunderstandings and ambivalent feelings about the benefits of amplification (Fitzpatrick et al, 2016), and are consistent with findings reported from other research with parents (McCracken et al, 2008; Muñoz et al, 2014). Taken together, these results suggest that when amplification is prescribed, an upfront investment may be worthwhile. In particular, our study suggests that parents of children with unilateral hearing loss require considerable support to use amplification as one-third (11 of 30) did not acquire or use amplification despite the audiological recommendation. When combined with inconsistent use, this means that more than half of the children with unilateral loss did not achieve ongoing use of their recommended amplification.

Given the apparent amount of time invested in managing these children, future research specifically geared toward achieving a better understanding of the benefits and costs of amplification and how to support families in their use of devices seems warranted. As pointed out by Moeller et al (2009), improved understanding of the factors that present challenges for hearing aid use can help shape the type of information and guidance provided to parents. Given that up to $40-50 \%$ of young children are now first identified with minimal loss and prescribed amplification, it seems that investing in improving our understanding specifically of factors affecting these families' ability to use amplification would be worthwhile. This is of particular importance as many children may not receive or require regular long-term language intervention sessions like children with more severe loss, whose parents receive ongoing guidance.

\section{CONCLUSIONS}

$T$ his study indicates that audiologists now routinely recommend amplification for children with minimal hearing loss at an early age. However, parent report points to the challenge of establishing consistent amplification use, as only two-thirds of all children and less than half of those with unilateral loss reportedly wore their devices on an ongoing basis. Given that very few children increased amplification use throughout the study, our findings suggest that the groundwork for improving auditory access through amplification needs to be laid at the outset if there is an expectation for auditory benefits from amplification in children with minimal loss, particularly those with unilateral loss. Although many children may not be enrolled in specific spoken language intervention, these results highlight the need for intervention that provides some level of specific parent support to establish amplification use in the early stages of the care process.

Acknowledgments. We are grateful to the families who participated in this research and to the collaborating institutions which provided parents with information about the study, assisted with ethics requirements, and provided audiological information.

\section{REFERENCES}

American Academy of Audiology (AAA). (2013) Clinical Practice Guidelines: Pediatric Amplification. http:/galster.net/wp-content/ uploads/2013/07/AAA-2013-Pediatric-Amp-Guidelines.pdf. Accessed January 5, 2017.

Bagatto M, Moodie S, Brown C, Malandrino A, Richert F, Clench D, Scollie S. (2016) Prescribing and verifying hearing aids applying the American Academy of Audiology pediatric amplification guideline: protocols and outcomes from the Ontario Infant Hearing Program. J Am Acad Audiol 27(3):188-203.

Bagatto MP, Tharpe AM. (2014) Decision support guide for hearing aid use in infants and children with minimal/mild bilateral hearing loss. In: Northern J, ed. A Sound Foundation Through Early Amplification 6th International Conference Proceedings. Stafa, Switzerland: Phonak AG.

Barreira-Nielsen C, Fitzpatrick E, Hashem S, Whittingham J, Barrowman N, Aglipay M. (2016) Progressive hearing loss in early childhood. Ear Hear 37(5):e311-e321.

Brown CL, Mackenzie S. (2005) The role of the audiologist and family support worker in the Ontario infant hearing program: a team approach. J Speech Lang Pathol Audiol 29:106-111. 
Davis A, Reeve K, Hind S, Bamford JM. (2001) Children with mild and unilateral hearing impairment. In: Seewald RC, Gravel JS, eds. A Sound Foundation through Early Amplification 2001: Proceedings of the Second International Conference. Suffolk, UK: St. Edmundsbury Press.

Durieux-Smith A, Fitzpatrick E, Whittingham J. (2008) Universal newborn hearing screening: a question of evidence. Int $J$ Audiol 47(1):1-10.

Fitzpatrick E, Grandpierre V, Durieux-Smith A, Gaboury I, Coyle D, Na E, Sallam N. (2016) Children with mild bilateral and unilateral hearing loss: parents' reflections on experiences and outcomes. J Deaf Stud Deaf Educ 21(1):34-43.

Fitzpatrick EM, Durieux-Smith A, Whittingham J. (2010) Clinical practice for children with mild bilateral and unilateral hearing loss. Ear Hear 31(3):392-400.

Fitzpatrick EM, Whittingham J, Durieux-Smith A. (2014) Mild bilateral and unilateral hearing loss in children: a 20 year view of hearing characteristics and audiologic practices before and after newborn hearing screening. Ear Hear 35:10-18.

Fitzpatrick EM, Roberts C, Whittingham J, Barreira-Nielsen C. (2017) Amplification decisions for children with mild bilateral and unilateral hearing loss. Can J Speech-Lang Pathol Audiol 41:203-226.

Ghogomu N, Umansky A, Lieu JE. (2014) Epidemiology of unilateral sensorineural hearing loss with universal newborn hearing screening. Laryngoscope 124(1):295-300.

Hyde A, Friedberg J, Price D, Weber S. (2004) Ontario infant hearing program: program overview, implications for physicians. Ont Med Rev January: 27-31.

Jones C, Feilner M. (2013) What do we know about the daily fitting and usage of hearing instruments in pediatrics? In: Seewald RC, Bamford JM, eds. A Sound Foundation through Early Amplification: Proceedings of the 2013 International Conference. Chicago, IL: Phonak AG.

Jones C, Launer S. (2011) Pediatric fittings in 2010: the sound foundations cuper project. In: Seewald RC, Bamford JM, eds. A Sound Foundation through Early Amplification: Proceedings of the 2010 International Conference. Chicago, IL: Phonak AG.

King AM. (2010) The national protocol for paediatric amplification in Australia. Int J Audiol 49(Suppl 1):S64-S69.

Martin P, Stroud J, Nicholson N. (2005) Hearing aids: helping parents understand the good, the bad and the ugly. Presentation at the $\mathrm{Na}$ tional EHDI Conference, Atlanta, GA. http://www.infanthearing.org/ meeting/ehdi2005/presentations/06-Martin_EHDI2005.ppt. Accessed January 5, 2017.

McCracken W, Young A, Tattersall H. (2008) Universal newborn hearing screening: parental reflections on very early audiological management. Ear Hear 29(1):54-64.

McKay S, Gravel JS, Tharpe AM. (2008) Amplification considerations for children with minimal or mild bilateral hearing loss and unilateral hearing loss. Trends Amplif 12(1):43-54.
Moeller MP, Hoover B, Peterson B, Stelmachowicz P. (2009) Consistency of hearing aid use in infants with early-identified hearing loss. Am J Audiol 18(1):14-23.

Muñoz K, Hill MM. (2015) Hearing aid use for children with hearing loss: a literature review. Perspect Hear Hear Disord Child 25(1):4-14

Muñoz K, Olson WA, Twohig MP, Preston E, Blaiser K, White KR. (2015) Pediatric hearing aid use: parent-reported challenges. Ear Hear 36(2):279-287.

Muñoz K, Preston E, Hicken S. (2014) Pediatric hearing aid use: how can audiologists support parents to increase consistency? $J$ Am Acad Audiol 25(4):380-387.

Muñoz K, Rusk SE, Nelson L, Preston E, White KR, Barrett TS, Twohig MP. (2016) Pediatric hearing aid management: parentreported needs for learning support. Ear Hear 37(6):703-709.

Ontario Ministry of Children and Youth Services (OMCYS). (2014) Bagatto M, Scollie S, eds. Ontario Infant Hearing Program Protocol for the Provision of Amplification, Version 2014.01. https:// www.mountsinai.on.ca/care/infant-hearing-program/documents/ ihp_amplification-protocol_nov_2014_final-aoda.pdf.

Peduzzi P, Concato J, Kemper E, Holford TR, Feinstein AR. (1996) A simulation study of the number of events per variable in logistic regression analysis. J Clin Epidemiol 49(12):1373-1379.

Porter H, Bess FH, Tharpe AM. (2016) Minimal hearing loss in children. In: Tharpe AM, Seewald R, eds. Comprehensive Handbook of Pediatric Audiology. 2nd ed. San Diego, CA: Plural Publishing, 887-914.

Reeve K. (2005) Amplification and family factors for children with mild and unilateral hearing impairment. In: National Workshop on Mild and Unilateral Hearing Loss: Workshop Proceedings. Brekenridge, CO: Centers for Disease Control and Prevention.

Tomblin JB, Walker EA, McCreery RW, Arenas RM, Harrison M, Moeller MP. (2015) Outcomes of children with hearing loss: data collection and methods. Ear Hear 36(Suppl 1):14S-23S.

Walker EA, Holte L, McCreery RW, Spratford M, Page T, Moeller MP. (2015) The influence of hearing aid use on outcomes of children with mild hearing loss. J Speech Lang Hear Res 58(5): 1611-1625.

Walker EA, McCreery RW, Spratford M, Oleson JJ, Van Buren J, Bentler R, Roush P, Moeller MP. (2015) Trends and predictors of longitudinal hearing aid use for children who are hard of hearing. Ear Hear 36(Suppl 1):38S-47S.

Walker EA, Spratford M, Moeller MP, Oleson JJ, Van Buren J, Bentler R, Roush P, Moeller MP. (2013) Predictors of hearing aid use time in children with mild-to-severe hearing loss. Lang Speech Hear Serv Sch 44(1):73-88.

Wood SA, Sutton GJ, Davis AC. (2015) Performance and characteristics of the newborn hearing screening programme in England: the first seven years. Int J Audiol 54(6):353-358. 